\title{
Blood-Brain Barrier Permeation of Glutathione-Coated Nanoparticle
}

\author{
Aditya Grover, Anjali Hirani, Vijaykumar Sutariya* \\ Department of Pharmaceutical Sciences, College of Pharmacy, University of South Florida, Tampa, FL 33612
}

Received: November 16, 2013; Accepted: January 17, 2014; Published: January 20, 2014

*Corresponding author: Vijaykumar Sutariya, Department of Pharmaceutical sciences, College of Pharmacy, University of South Florida, 12901 Bruce B. Downs Blvd. MDC 30Tampa, FL 33612-4749, Tel: 813-974-1401; Fax: 813-974-9890; E-mail: vsutariy@health.usf.edu

\begin{abstract}
Brain-related diseases are some of the most aggressive, but are also some of the hardest to treat. One of the main obstacles in attempting to treat brain-related diseases is surmounting the blood-brain barrier, a physiological barrier that separates neural tissue from the blood through the interactions of glial cells and endothelial cells. The present study describes the establishment of an in vitro blood-brain barrier model through the co-culture of rat brain endothelial (RBE4) and astrocytic (C6) cells on Transwell ${ }^{\text {TM }}$ permeable support inserts. Coating organic, drug-encapsulating nanoparticles with glutathione helps the nanoparticles cross the in vitro blood-brain barrier model better than identical concentrations of uncoated nanoparticles and the free drug solution. This provides the basis for conjugating nanoparticles or other drug vectors with glutathione in clinical settings to enhance the trans-blood-brain barrier permeation of brain-targeted therapies. The implementation of this method in therapies has the potential to influence therapies of brain-related disorders by specifically targeting neural tissue with therapeutic compounds.
\end{abstract}

Keywords: Blood-Brain Barrier; PLGA; Nanoparticles Glutathione; Transwell

\section{The blood-brain barrier}

The blood-brain barrier (BBB) is a physical interface in the central nervous system (CNS) that exists between the blood and neural tissue. The phenotype of the BBB is primarily the result of the interactions between three main cell types: Endothelial cells, astrocytes, and pericytes [1]. The interactions of the endothelial cells and astrocytes seems to be of utmost importance in maintaining the integrity of the $\mathrm{BBB}$, as close to $99 \%$ of the basement membrane is covered by astrocytes and the interaction of these 2 cell types maintains the expression of tight junctional proteins $[2,3]$. However, the physiology of the BBB prevents the permeation of water and certain compounds across the BBB into the brain [3]. This greatly limits the availability of drugs to the brain. The development of a drug delivery mechanism that would be able to permeate the BBB would greatly increase the amount of effective therapies against CNS-related diseases such as cancers, Parkinson's, and Alzheimer's, among others.

\section{Glutathione as a permeation-inducer}

Glutathione (GSH) is a tripeptide antioxidant that protects tissues from reactive oxidative species (ROS) and other types of oxidative damages [4]. GSH interacts with transmembrane proteins located in the brain that are involved in the active transport of certain substances across the BBB [4]. Among these, P-glycoprotein is involved in ATP-coupled reactions in transporting compounds conjugated to GSH across membranes; a number of other proteins are also involved in similar mechanisms $[5,4]$. Drug delivery systems conjugated with GSH provide for an interesting route of potential therapies directed towards the brain; the manipulation of existing GSH-transport mechanisms may be utilized to enhance the trans-BBB permeation of therapeutic compounds. The great number of GSH receptors that are present in the CNS may be an advantage in targeting these receptors to mediate the active or passive transcytosis of therapeutic compounds $[6,7]$.

\section{Establishment of a Transwell ${ }^{\mathrm{TM}}$ co-culture}

To compare the permeation of GSH-coated nanoparticles to the free drug solution, an in vitro BBB model was established by modifying a preexisting protocol [8] Transwell ${ }^{\mathrm{TM}}$ permeable inserts (Corning ${ }^{\mathrm{TM}}$, No. 3401) with $0.4 \mu \mathrm{m}$ sized pores were purchased upon which to seed the cellular co-culture on either side of the permeable insert. The $0.4 \mu \mathrm{m}$ sized pores allow the sharing of cellular factors between the co-culture to induce BBB-specific cellular characteristics in endothelial cells, such as tight junctions, without the physical transfer of cells. In addition, the size of pores is large enough to allow the passage of nanoparticles ; a size of $150 \mathrm{~nm}$ is ideal for trans-BBB permeation [9]. Both sides of the Transwell ${ }^{\mathrm{TM}}$ insert were coated with $0.1 \%$ rat tail collagen I (BD ${ }^{\mathrm{TM}}$ Biosciences). C6 cells (rat astrocytoma cell line) was seeded to the bottom side of the insert, and RBE4 (immortalized rat brain endothelial cell line) was seeded on the inside of the insert (Figure 1). It was hypothesized that the transfer of astrocytic growth factors from the C6 cell line across the porous membrane would induce the formation of tight junctions in the RBE4 cell line on the other side of the insert, as is present in in vivo situations [2]. The Transwell ${ }^{\mathrm{TM}}$ co-culture 


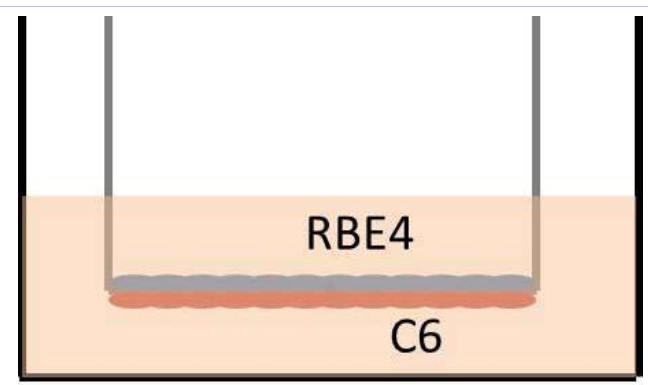

Figure 1: Graphical representation of blood-brain barrier model established using Transwell ${ }^{\mathrm{TM}}$ permeable supports. RBE4 rat brain endothelial cells are seeded inside the insert and C6 rat astrocytic cells are seeded on the bottom side of the insert. Both are bathed in the same 1:1 mixture of Ham's F10:MEM media. $2 \mathrm{~mL}$ of total media is used, $1.5 \mathrm{~mL}$ media is under the support and $0.5 \mathrm{~mL}$ is in the support.

was bathed in $2 \mathrm{~mL}$ of media, 1.5 of which was around the insert and 0.5 of which was inside the insert. The media used was a 1:1 mixture of Ham's F10: MEM (Cellgro ${ }^{\mathrm{TM}}$ ), supplemented with $10 \%$ fetal bovine serum (FBS, Invitrogen ${ }^{\mathrm{TM}}$ ), 1\% streptomycin and penicillin (Invitrogen ${ }^{\mathrm{TM}}$ ), human recombinant diluted basic fibroblast growth factor (bFGF) (BD Biosciences ${ }^{\mathrm{TM}}$ ), and HEPES buffer (Fisher $^{\mathrm{TM}}$ ). The cells were grown in an incubator in standard humidity conditions and $37^{\circ}$ Celsius and $5 \% \mathrm{CO}_{2}$. Identical, biologically significant concentrations of the free drug solution and GSH-coated nanoparticles were added to the $0.5 \mathrm{~mL}$ media that bathed the inside of the Transwell ${ }^{\mathrm{TM}}$ insert. Samples of the media $(100 \mu \mathrm{L})$ from underneath the permeable support ("across" the BBB) were taken at predetermined intervals and replaced with fresh media. The concentration of the permeated drug solution and GSH-coated nanoparticle was determined by UV spectroscopy at wavelengths specific to the drug by being compared to standard dilutions of the drug also analyzed by UV spectroscopy at the same wavelength.

\section{BBB permeation of GSH-conjugated nanoparticles}

Using a Transwell ${ }^{\mathrm{TM}}$ based BBB model, we were able to show that GSH-coated PEGylated PLGA nanoparticles were able to permeate a BBB model better than a free drug solution by using fluorescence spectroscopy to quantify the permeation of doxorubicin, and UV-vis absorbance to quantify the permeation of methylene blue.

Two different drugs, methylene blue (MB) and doxorubicin (Dox) were encapsulated by PEGylated PLGA nanoparticles using a previously reported method [7]. Briefly, $4 \mathrm{mg}$ of each of the drugs were dissolved in $3 \mathrm{~mL}$ of acetone; $120 \mathrm{mg}$ of PLGAPEG-COOH polymer (Lakeshore Biomaterials ${ }^{\mathrm{TM}}$ ) was added to the acetone. The resultant solution was added dropwise to 10 $\mathrm{mL}$ of deionized water, spinning at 300 RPM with a magnetic stirring bar and at $40^{\circ}$ Celsius. The nanoparticles were allowed to precipitate out and the acetone was allowed to evaporate completely. The resultant nanoparticle suspension was collected in a $15 \mathrm{~mL}$ conical flask and centrifuged (3000 RPM, 10 minutes) to collect all the nanoparticles. The supernatant was discarded and replaced with fresh deionized water to make up a total volume of $10 \mathrm{~mL}$.

The characterization of the resultant nanoparticles revealed that the average size of the Dox nanoparticles to be $99.8 \mathrm{~nm}$ $\pm 2.58 \mathrm{~nm}$ with an entrapment efficiency of $72.4 \% \pm 6.14 \%$. Characterization of the resultant MB nanoparticles yielded an average size of $150 \mathrm{~nm} \pm 10 \mathrm{~nm}$ with an entrapment efficiency of $25.07 \% \pm 3.19 \%$. The entrapment efficiency of each nanoparticle formulation was used to determine the amount of nanoparticle suspension to be added to achieve equal amounts of free drug solution and nanoparticle suspension.

Before treating the cells in the Transwell ${ }^{\mathrm{TM}}$ inserts, $20 \mathrm{mg}$ of GSH was added to $1 \mathrm{~mL}$ of each of the nanoparticle solutions to yield a 2\% GSH coat on the particles, which refers to the amount of GSH per $1 \mathrm{~mL}$ of nanoparticle suspension, not a weightdependent measurement [7].

Figures $2 \mathrm{~A}$ and $-\mathrm{B}$ show the comparative permeation of the free drug solution with the respective drug-encapsulated, GSHcoated nanoparticle 24 hours after treatment.

Figure 2A represents the comparative permeability data of $1 \mu \mathrm{M}$ each Doxfree drug solution, uncoated Dox-encapsulated nanoparticles, and GSH-coated Doxnanoparticles 24 hours after treatment as quantified by fluorescent spectroscopy (Synergy H4 plate reader, Biotek Instruments ${ }^{\mathrm{TM}}$ ) with an excitation wavelength of $470 \mathrm{~nm}$ and emission wavelength of $590 \mathrm{~nm}$. As can be seen by the data, there is a 1.3 -fold greater permeation

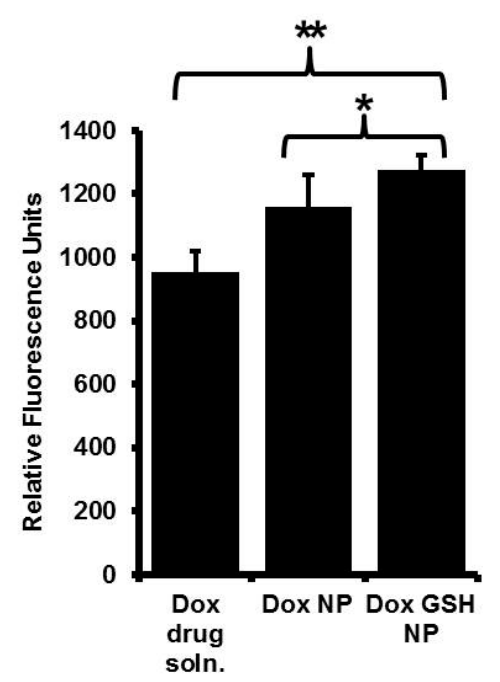

Figure 2a: The comparative permeation of $1 \mu \mathrm{M}$ free Dox drug solution, an equal concentration of uncoated Dox-encapsulated nanoparticle, and GSH-coatedDox nanoparticle across the Transwell ${ }^{\mathrm{TM}}$ co-culture of RBE4 and C6 cells after 24 hours of treatment. Sample of media removed from under the support was analyzed by fluorescence spectroscopy (excitation wavelength, $470 \mathrm{~nm}$ and emission wavelength, $590 \mathrm{~nm}$ ). Readings of duplicates were taken 3 times and averaged $(n=6)$ and standard deviation was taken from data. T-test was used to statistically analyze the data $(\mathrm{p}<0.05)$. 
of the drug-encapsulated, GSH-coated nanoparticles as compared to the free drug solution $(n=3)$. There is a significant increase in the permeation of the GSH-coated nanoparticle as compared to the free drug solution and uncoated Dox nanoparticle ( $p<$ 0.05). After performing MTT assay in RG2 cells with Dox drug solution, uncoated nanoparticles, and GSH-coated nanoparticles, it was shown that the uncoated nanoparticles and GSH-coated nanoparticles were less toxic to the cells than the free drug solution after 24 hours (data not shown).

Similarly, Figure 2B represents the comparative permeability data of MB free drug solution, uncoated MB-encapsulated nanoparticles, and GSH-coated MB nanoparticles 24 hours after treatment as quantified by UV-visual spectroscopy (Synergy $\mathrm{H} 4$ plate reader, Biotek Instruments ${ }^{\mathrm{TM}}$ ) at a wavelength of 600 $\mathrm{nm}$. The data represents a 1.5-fold greater permeation of MBencapsulated, GSH-coated nanoparticles as compared to the free drug solution across the in vitro BBB model $(n=3)$.There is a statistically significant increase in the permeation of the GSH coated MB nanoparticle as opposed to the uncoated nanoparticle and free drug solution $(p<0.05)$.The data suggests that GSHcoated nanoparticles may be able to be used clinically to permeate the BBB for therapeutic purposes

\section{Conclusion and Future Perspectives}

Permeation data comparing the free drug solution with drug-encapsulated nanoparticles for two brain-targeted drugs revealed an increased permeation of GSH-coated nanoparticles as compared to the free drug solution in a Transwell ${ }^{\mathrm{TM}}$ co-culture of rat brain endothelial and astrocytoma cells. The Transwell ${ }^{\mathrm{TM}}$ apparatus is a great way to investigate the physiology of the BBB in a controlled, in vitro setting. A cellular monoculture of brain

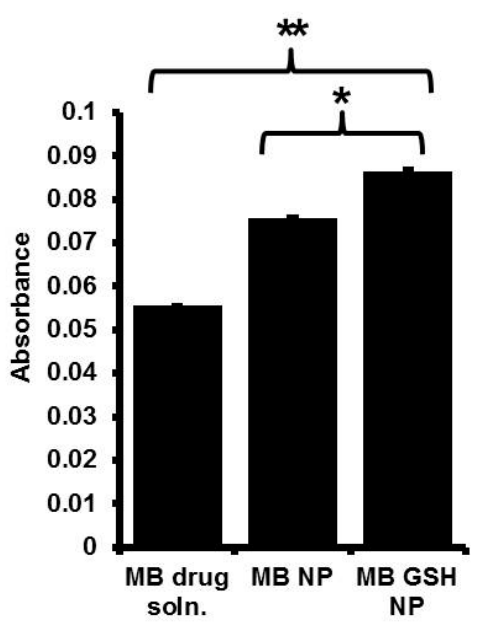

Figure 2b: The comparative permeation of $10 \mu \mathrm{M}$ free $\mathrm{MB}$ drug solution, an equal concentration of uncoated MB-encapsulated nanoparticles, and GSH-coated MB nanoparticle across the Transwell ${ }^{\mathrm{TM}}$ co-culture of RBE4 and C6 cells after 24 hours of treatment. Sample of media removed from under the support was analyzed by UV-vis spectroscopy (wavelength, $600 \mathrm{~nm}$ ). Readings were taken 3 times and averaged $(n=3)$ and standard deviation was taken from data. T-test was used to statistically analyze the data $(\mathrm{p}<0.05)$. endothelial cells may not be able to express the necessary tight junctions that are characteristic of the BBB. Because the culturing of astrocytes with endothelial cells is seem as "highly necessary" in inducing BBB-specific properties [10], the Transwell ${ }^{\mathrm{TM}}$ system is a great asset to investigating trans-BBB permeabilities.

Although the Transwell ${ }^{\mathrm{TM}}$ model provides a co-culture of the two main cell types that interact at the BBB, the interaction of pericytes with endothelial cells should also be investigated. Both, pericytes and astrocytes are glial cells and it is these types of cells that are responsible for inducing the expression of BBB-specific properties in endothelial cells [11]. It would also be interesting to investigate the co-culture of endothelial cells with pericytes. Interestingly, culturing endothelial cells solely in media from astrocytic cells is useful in inducing BBB-specific physiological characteristics in the endothelial cells [12]. Perhaps a co-culture of endothelial cells and pericytes bathed in astrocytic medium would also be able to induce BBB-specific characteristics in endothelial cells.

Recently, there have been developments in the establishment of co-cultures for in vitro BBB models. Matrigel (Cultrex or EHS matrix) is a basement membrane-like, three-dimensional gel upon which cells can be seeded to act in three-dimensional ways [13]. Matrigel could be used to grow co-cultures of endothelial cells, astrocytes, and pericytes. The three-dimensional interaction of these three cell types may cause the exhibition of characteristics that are not usually visible in other in vitro settings and may be a better indicator of the in vivo setting [13]. A similar co-culturing method is the Spheroidal method. In this model, co-cultures of multiple cell types can be seeded onto a membrane from which the spheroidal culture can be detached [14]. This model may also be able to shed light on cellular interactions that are present in three-dimensional settings. Although these models show promises in investigating BBB properties, their relatively recent development as compared to the Transwell ${ }^{\mathrm{TM}}$ system requires more usage to be as established as the Transwell ${ }^{\mathrm{TM}}$ system which is considered to be the "gold standard" when investigating BBBrelated permeability [15].

The permeation data that is presented in this work shows evidence that GSH-coated nanoparticles are able to better permeate the BBB than the free drug solution. We have previously investigated the permeation of GSH-coated paclitaxel nanoparticles in an in vivo model and showed that the GSHcoated nanoparticles are able to permeate the BBB in mouse models [7]. Greater emphasis should be placed on such a model in the development of brain-targeted therapies. GSH-coated nanomedicines would greatly benefit clinical obstacles to brain therapeutics and may be able to increase the life-expectancy and quality of life of millions suffering from similar diseases.

\section{References}

1. Imola W, Csilla F, Istvan KA (2011) In vitro models of the blood-brain barrier. Acta Neurobiol Exp 71: 113-128.

2. Abbott NJ (2002) Astrocyte-endothelial interactions and blood-brain barrier permeability. J Anat 200: 629-638. 
3. Li Guanglei, Simon Melissa J, Cancel Limary M, Shi Zhong-Dong, Ji Xinying, et al. (2010) Permeability of endothelial and astrocyte cocultures: in vitro blood-brain barrier models for drug delivery studies. Ann Biomed Eng 38: 2499-2511.

4. Smeyne M, Smeyne RJ (2013) Glutathione metabolism and parkinson's disease. Free Radio Biol Med 62: 13-25.

5. Bellarosa C, Bortolussi G, Tiribelli C (2009) The role of ABC transporters in protecting cells from bilirubin toxicity. Curr Pharma Des 15: 2884-2892.

6. Gaillard PJ (2010) glutathione-based drug delivery system: WO patent $2,010,095,940 \mathrm{~A} 2$.

7. Geldenhuys W, M bimba T, Bui T, Harrison K, Sutariya V (2011) Braintargeted delivery of paclitaxel using glutathione-coated nanoparticles for brain cancers. J Drug Target 19: 837-845.

8. Etame AB, Smith CA, Chan WC, Rutka JT (2011) Design and potential application of PEGylated gold nanoparticles with size-dependent permeation through brain microvasculature. Nanomedicine: Nanotechnology, Biology and Medicine 7: 992-1000.

9. Carroll RT, Bhatia D, Geldenhuys W, Bhatia R, Miladore N, et al
(2010) Brain-targeted delivery of Tempol-loaded nanoparticles for neurological disorders. J Drug Target 18: 665-674.

10. Cecchelli R, Dehouck B, Descamps L, Fenart L, Bu ee-Scherrer V, et al. (1999) In vitro model for evaluating drug transport across the bloodbrain barrier. Adv drug deliv rev 36: 165-178.

11. Naik P, Cucullo L (2012) In vitro blood-brain barrier models: current and perspective technologies. J pharma sci 101: 1337-1354.

12. Cecchelli R, Berezowski V, Lundquist S, Culot M, Renftel M, et al. (2007) Modelling of the blood-brain barrier in drug discovery and development. Nat Rev Drug Discov 6: 650-661.

13. Kleinman HK, Martin GR (2005) Matrigel: basement membrane matrix with biological activity. Semin Cancer Biol 15: 378-386.

14. Hirschhaeuser F, Menne H, Dittfeld C, West J, Mueller-Klieser W, et al. (2010) Multicellular tumor spheroids: an underestimated tool is catching up again. J Biotechnol 148: 3-15.

15. Hatherell K, Couraud PO, Romero IA, Weksler B, Pilkington GJ (2011) Development of a three-dimensional, all-human in vitro model of the blood-brain barrier using mono-, co-, and tri-cultivation Transwell models. J Neurosci Methods 199: 223-229. 